\title{
DERECHO Y POLÍTICAS AMBIENTALES EN EL PAÍS VASCO (SEGUNDO SEMESTRE 2019)
}

\author{
IÑIGO LAZKANO BROTÓNS \\ Profesor colaborador \\ Universidad del País Vasco / Euskal Herriko Unibersitatea
}


Sumario: 1. Introducción. 2. Novedades legislativas con incidencia ambiental sobre patrimonio cultural y ordenación de grandes establecimientos comerciales. 3. Las nuevas Directrices de Ordenación del Territorio del País Vasco. 4. Desarrollo normativo en materia de residuos. 5. Desarrollo de la política de espacios protegidos. 6 . Zonas sensibles en las cuencas internas y en las aguas marítimas. 7. Ruido de espectáculos públicos y actividades recreativas. 8. Actividad de fomento ambiental.

\section{INTRODUCCIÓN}

Tres son las novedades normativas más importantes del período que abarca la presente crónica. En primer lugar, aunque no desde el punto de vista del rango normativo, la aprobación de unas nuevas Directrices de Ordenación del Territorio del País Vasco, en sustitución de las adoptadas en 1997. Por otra parte, se han aprobado dos leyes con evidentes implicaciones ambientales, aunque más desde la perspectiva de la planificación territorial: la reguladora del patrimonio cultural y la de ordenación territorial de grandes establecimientos comerciales.

Desde el punto de vista de la actividad legislativa, también merece mencionarse el hecho de que se ha sometido a información pública el anteproyecto de Ley de Cambio Climático de Euskadi (Resolución de 4 de junio de 2019, del Director de Patrimonio Natural y Cambio Climático, BOPV 110, 12 de junio).

\section{NOVEDADES LEGISLATIVAS CON INCIDENCIA AMBIENTAL SOBRE} PATRIMONIO CULTURAL $Y$ ORDENACIÓN DE GRANDES ESTABLECIMIENTOS COMERCIALES

Se ha aprobado la Ley 6/2019, de 9 de mayo, de Patrimonio Cultural Vasco (BOPV 93, 20 de mayo). El objeto de esta ley (en adelante LPCV) es establecer el régimen jurídico de dicho patrimonio, con el fin de garantizar su protección, conservación y puesta en valor. Evidentemente en la norma se abordan cuestiones que van más allá de las propiamente ambientales y territoriales, pero también hay que considerar las afecciones que en esos ámbitos produce la ley, puesto que dentro de los bienes del patrimonio cultural vasco (que pueden ser inmuebles, muebles e inmateriales) se incluyen, por ejemplo, los que ostentan un valor paleontológico o paisajístico. Así, por ejemplo, cuando se procede a la declaración de protección de un bien cultural, la resolución debe incluir entre sus contenidos mínimos "la relación de elementos degradantes, incluidos los 
causantes de contaminación visual o acústica, para su atenuación o eliminación" (art. 16.d LPCV).

La incidencia más fuerte de la LPCV en materia medioambiental se observa, precisamente, al regular el régimen específico de protección de los bienes culturales inmuebles. Más allá de algunas reglas generales que son habituales en la ordenación de este tipo de sectores (por ejemplo, la preceptividad de la autorización sectorial previa al otorgamiento de licencias urbanísticas sobre bienes culturales declarados, la regulación del carácter excepcional de los desplazamientos de bienes culturales inmuebles o la necesidad de informe favorable del órgano sectorial en materia de patrimonio cultural sobre todo tipo de instrumentos de ordenación que afecten a bienes culturales declarados), hay que destacar tres previsiones específicas:

a) La protección otorgada por la LPCV a los bienes inmuebles inscritos en el registro del patrimonio cultural vasco, así como a los incluidos en las zonas de presunción arqueológica, "prevalecerá sobre los instrumentos de ordenación urbanística, territorial y medioambiental" que dispondrán de un plazo máximo de dos años para su adaptación (art. 47.2).

b) En la tramitación de todas las evaluaciones de impacto ambiental que puedan afectar directa o indirectamente a los bienes culturales, así como a las zonas de presunción arqueológica, la Administración competente en materia de medio ambiente solicitará informe del departamento del Gobierno Vasco competente en materia de patrimonio cultural, que tendrá carácter preceptivo y vinculante, debiendo incluirse sus determinaciones en la declaración ambiental (art. 47.5)

c) Se establecen reglas destinadas a prohibir y eliminar la instalación de elementos que originen contaminación visual o acústica sobre los bienes culturales protegidos, dificultando o distorsionando su contemplación (art. 50).

El significado de la Ley 10/2019, de 27 de junio (BOPV 129, 9 de julio), de ordenación territorial de grandes establecimientos comerciales (en adelante LOTGEC), viene determinado por su origen. El Tribunal Supremo declaró la nulidad, por diversas sentencias de 2015, de varias determinaciones que imponían limitaciones a la superficie máxima para equipamientos comerciales mediante el plan territorial sectorial de creación pública de suelo para actividades 
económicas y equipamientos comerciales. Tales restricciones, señalaban las decisiones judiciales, debían estar suficientemente motivadas en una norma con rango de ley. Además, la introducción de limitaciones superficiales a su implantación no podía obedecer a razones exclusivamente comerciales y mercantiles y, en todo caso, cualquier restricción debía observar la triple condición de no discriminación, necesidad y proporcionalidad.

La LOTGEC va a regular, en consecuencia, la implantación y ampliación de los grandes establecimientos comerciales (que se definen en el propio texto legal combinando los usos a los que están destinados con su edificabilidad urbanística, edificabilidad que varía en función del tipo de municipios en los que se enclaven). La ley descansa en varios principios: la libertad de empresa, la libertad de establecimiento -con las limitaciones admisibles a la misma-, la cooperación y coordinación interadministrativa y el principio de desarrollo sostenible (art. 4). Estos principios están orientados a la consecución de diversos objetivos que se explicitan en la ley (art. 5), varios de los cuales son evidentemente ambientales: la ocupación sostenible del suelo y la preservación de sus valores; la protección de los entornos y del medio ambiente urbano, rural y natural; la minimización del impacto de la implantación de tales establecimientos sobre el territorio; el robustecimiento de un modelo de ciudad compacta; el estímulo de la movilidad sostenible mediante el fortalecimiento de las redes de transporte público y colectivo; la reducción de los desplazamientos en modos de transporte privado que contribuyen a la producción de emisiones contaminantes a la atmósfera; la preservación del paisaje, rural y urbano, y del patrimonio cultural y natural, así como el fomento de la biodiversidad urbana; etc. Para alcanzar tales fines la LOTGEC establece las condiciones de implantación de los grandes establecimientos comerciales, regulándose generalmente su ubicación (siempre que no tengan "carácter singular") en la trama urbana residencial, aunque con algunas excepciones derivadas de la continuidad y colindancia con otros ámbitos de ordenación no residenciales -pero comprendidos en la malla urbana- $y$, en cualquier caso, con límites cuantitativos mínimos de perímetro colindante o máximos de superficie y edificabilidad. También se regulan con cierto detalle las condiciones de ampliación de los grandes establecimientos ya existentes. 


\section{LAS NUEVAS DIRECTRICES DE ORDENACIÓN DEL TERRITORIO DEL PAÍS VASCO}

Por Decreto 128/2019, de 30 de julio (BOPV 181, 24 de septiembre), se han aprobado definitivamente las Directrices de Ordenación Territorial del País Vasco (DOT). Estas nuevas DOT sustituyen a las precedentes de 1997, tratando de abordar desde el mismo vértice de la planificación territorial los retos derivados del actual contexto ambiental, económico y sociodemográfico (el cambio climático, la opción por la economía circular, el estancamiento poblacional y su envejecimiento, la terciarización de la industria, etc.). El Decreto consta de cinco artículos, dos disposiciones adicionales, cuatro transitorias, una derogatoria y una final. Como dice la Ley de Ordenación del Territorio del País Vasco, y asume el art.3 del Decreto, las DOT están integradas por tres elementos:

a) Memoria, que consta de doce apartados y que no se publica en el BOPV, sino que aparece referenciada en la página web del departamento competente en la materia del Gobierno Vasco (el Departamento de Medio Ambiente, Planificación Territorial y Vivienda: DMAPTV). En esta Memoria se incluyen como temas: 1) el marco de la revisión, 2) las bases y principios rectores del modelo territorial, 3) los elementos definitorios del modelo territorial, 4) el medio físico y la infraestructura verde, 5) el hábitat rural, 6) el hábitat urbano, 7) el paisaje, el patrimonio cultural y natural y los recursos turísticos, 8) la gestión sostenible de los recursos (agua, energía y economía circular), 9) la movilidad y logística, 10) las cuestiones transversales (accesibilidad universal, perspectiva de género, cambio climático, salud, euskera e interrelación territorial), 11) la gobernanza, y 12) anexos sobre la infraestructura verde, los servicios de los ecosistemas y los indicadores de sostenibilidad territorial y urbanística.

b) Mapa Síntesis, que, como en el caso anterior, no aparece en el BOPV sino solo en su versión en la web referida.

c) Normas de aplicación, que figuran como Anexo I del Decreto 128/2019 y que se publican íntegramente en el BOPV. Estas normas de aplicación se estructuran en tres capítulos (I. Objeto y Naturaleza. II. Directrices de 
Ordenación y Uso del Espacio. III. Directrices Recomendatorias), cinco anexos (I. Identificación de la eficacia vinculante directa o indirecta de las directrices del capítulo II. II. Ordenación del Medio Físico. III. Municipios que componen cada Área Funcional. IV. Cuantificación Residencial. V. Normativa sectorial introducida de acuerdo con los informes de la Administración Central), tres disposiciones adicionales, cuatro transitorias, una derogatoria y una final.

Además, el Decreto 128/2019 cuenta con un Anexo II, en el que se incluye el extracto relativo a la integración de los aspectos ambientales, incluidas las medidas de seguimiento de los efectos en el medio ambiente, a los efectos de lo previsto en la normativa de evaluación ambiental.

Como puede comprobarse a primera vista, el catálogo de cuestiones y reglas medioambientales que se abordan en las DOT es amplio, y es imposible hacer en este momento una detallada referencia del mismo. Pero hay que destacar ciertos elementos contenidos en las DOT, algunos importantes por contraste con lo que establecían las anteriores de 1997.

La nuevas DOT clasifican con más claridad que antes las normas de aplicación, diferenciando con nitidez cuáles son simples recomendaciones (las del Capítulo III del Anexo I) y cuales son estrictamente vinculantes (las del Capítulo II del Anexo I), distinguiendo dentro de éstas las que tienen eficacia directa e indirecta (estas últimas, las dirigidas a las diferentes Administraciones y que tienen que ser desarrolladas por el subsiguiente planeamiento territorial y urbanístico). Sin embargo, se detecta un problema grave que deberá ser resuelto. La disposición transitoria primera del Decreto 128/2019 establece que los planes territoriales parciales y los planes territoriales sectoriales han de adaptarse a las DOT en un plazo de ocho años (apartado 1), y que "continuarán vigentes en tanto no se produzca su adaptación" (apartado 4). Si se interpreta este último precepto de manera literal, no podría afirmarse la eficacia directa de las normas vinculantes que establece las DOT, pues todas serían de eficacia indirecta (al no ser aplicables hasta que su contenido se incorporase a un plan de los señalados), lo que no parece demasiado razonable. Hay que pensar, en consecuencia, que las directrices vinculantes de ordenación y uso del espacio catalogadas como de eficacia directa, al expresarse en normas concretas de aplicación general, son 
aplicables desde la entrada en vigor de las mismas DOT, sin necesidad de su incorporación a planeamiento alguno. Por eso resulta de especial interés cuales de ellas introducen novedades sustanciales en el sistema de ordenación vigente (pues otras muchas solo reproducen lo que ya existía con anterioridad).

Si se observa la matriz de ordenación del medio físico, en la que se recoge la ordenación del suelo no urbanizable de la CAPV que realiza las DOT, los cambios son escasos y poco relevantes, más allá de una mejor y más precisa definición tanto de los tipos de suelo como de los usos propiciados, admisibles o prohibidos. Desaparece uno de los tipos de suelo no urbanizable previstos en las directrices de 1997 (el denominado "sin vocación de uso definido") sin que se establezca cuál es el destino de las más de veintidós mil de hectáreas así clasificadas en los diversos planes urbanísticos locales. Desaparece en las nuevas DOT la limitación del carácter vinculante de la matriz a los casos del suelo no urbanizable de especial protección y de protección de aguas superficiales, por lo que puede afirmarse que en el nuevo modelo de ordenación establecido todas las previsiones de la matriz resultan vinculantes (y la mayor parte de las mismas de eficacia directa). Se establece también, como norma vinculante de eficacia directa, que en los casos en los que se dé una concurrencia de la llamada infraestructura verde (espacios protegidos, corredores ecológicos, humedales, etc.) con la infraestructura "gris" (infraestructuras viarias, desarrollos urbanos, etc.), prevalecerá la primera en aras a la realización de las tareas necesarias de restauración ecológica.

Otros aspectos que muestran una cierta tensión en las directrices son las previsiones que, simultáneamente, pretenden impulsar una cierta densificación de las zonas urbanas (a favor del modelo de ciudad compacta, estableciendo, por ejemplo, un perímetro de crecimiento urbano a los municipios) compatible con la permeabilización y continuidad de sus espacios libres; o, uno de los aspectos más importantes de las directrices, la fijación de criterios de cuantificación residencial que limite los desarrollos urbanos locales, si bien con una cierta flexibilidad pues los coeficientes fijados en las directrices pueden ser modificados por el Gobierno Vasco reglamentariamente.

Significativas son también las previsiones sobre equipamientos comerciales (aunque, en este sentido, prevalecerán por razones de rango normativo las 
previsiones de la citada LOTGEC) y la inclusión de ciertas reglas sobre incrementos residenciales en núcleos rurales, que desarrollarían aspectos inicialmente no previstos en la legislación urbanística vasca. En la regulación sobre actividades extractivas en suelo no urbanizable señalan las DOT que, en relación a la fractura hidráulica o "fracking", la incertidumbre sobre sus impactos, unida a la actuación en un territorio significativamente denso en cuanto a población y estructuras urbanas como la CAPV, desaconsejan su desarrollo.

\section{DESARROLLO NORMATIVO EN MATERIA DE RESIDUOS}

Por la conflictividad (política y judicial) que la materia produce, y aun no tratándose de una norma autonómica sino foral, hay que destacar en esta materia la aprobación de la Norma Foral 6/2019, de 20 de marzo, por la que se aprueba el Plan Integral de Gestión de Residuos Urbanos de Gipzukoa 20192030 (BOPV 79, 26 de abril).

Por otro lado, mediante Decreto 64/2019, de 9 de abril (BOPV 80, 30 de abril), se establece el régimen jurídico aplicable a las actividades de valorización de escorias negras procedentes de la fabricación de acero en hornos de arco eléctrico. Este reglamento pretende innovar la regulación preexistente, de 2003, a las nuevas exigencias e implicaciones derivadas del concepto de "economía circular" impulsado por la UE. Así, se categorizan, siempre bajo la consideración de criterios ambientales, varios tipos de usos contemplados para la utilización de áridos siderúrgicos (sometidos a autorización específica del órgano ambiental de la CAPV). Recibe una particular atención el empleo de este tipo de áridos en aplicaciones constructivas y en la fabricación de cemento, por la potencial afección ambiental ligada a una posible liberación de sus componentes. Pero la utilización final de las escorias valorizadas debe ajustarse también a los requisitos técnicos de carácter constructivo y a los demás que pudieran resultar de aplicación en función del destino final propuesto, por lo que no se excluye, en su caso, el pronunciamiento de otros órganos competentes en la materia.

También hay que informar de la aprobación del Decreto 63/2019, de 9 de abril, (BOPV 74, 16 de abril), por el que se establece el régimen jurídico y las condiciones técnicas de las instalaciones y actividades de compostaje comunitario. 


\section{DESARROLLO DE LA POLÍTICA DE ESPACIOS PROTEGIDOS}

Se ha procedido a la aprobación provisional, por parte del Consejero de MAPTV, de los planes de ordenación de recursos naturales de los espacios naturales protegidos de Gorbeia, Izki, Valderejo-Sobrón-Sierra de Arcena, Urkiola (Órdenes de 2 de abril de 2019, BOPV 74, 16 de abril) y de Armañón (Orden de 1 de junio de 2019, BOPV 135, 17 de junio).

Asismismo se ha sometido a información pública el proyecto de Decreto por el que se modifica el Plan Rector de Uso y Gestión de la Reserva de la Biosfera de Urdaibai (Resolución de 23 de julio de 2019, BOPV 146, 2 de agosto).

\section{ZONAS SENSIBLES EN LAS CUENCAS INTERNAS Y EN LAS AGUAS MARÍTIMAS}

Mediante el Decreto 111/2019, de 16 de julio (BOPV 142, 29 de julio) se han declarado las zonas sensibles en las cuencas internas y en las aguas marítimas de la CAPV, en aplicación de la normativa comunitaria y de la básica estatal sobre tratamiento de las aguas residuales urbanas. Esta normativa establece que la declaración debe revisarse al menos cada cuatro años, aunque la última vez en la que se efectuó esta declaración en la CAPV fue en 2012 (hace más de seis años, por tanto). Las concretas zonas son fijadas en el anexo al Decreto 111/2019: se trata de cuatro embalses (Aixola, Urkulu, Ibaiedere y Barrendiola) y siete estuarios (de los ríos Butroe, Oka, Lea, Artibai, Inurritza, Oiartzun y Bidasoa). Se regulan en la norma los tratamientos más rigurosos de los vertidos en las aglomeraciones afectadas. En la consideración del nutriente que debe ser reducido mediante tratamiento adicional podrá tenerse en cuenta lo siguiente: a) en los cursos de agua que desemboquen en embalses conviene prever, con carácter general la eliminación de fósforo; b) en los estuarios, bahías y otras aguas marítimas que tengan un intercambio de aguas escaso deberá preverse, con carácter general, la eliminación de nitrógeno. Las autorizaciones de vertidos podrán imponer requisitos más rigurosos cuando ello sea necesario para garantizar que las aguas receptoras cumplan con las normas de calidad ambiental fijadas en la normativa vigente y con los objetivos medioambientales que establezca el Plan Hidrológico de la demarcación. 


\section{RUIDO DE ESPECTÁCULOS PÚBLICOS Y ACTIVIDADES RECREATIVAS}

Por Decreto 119/2019, de 23 de julio (BOPV 142, 29 de julio) se ha procedido a la modificación del reglamento de desarrollo de la Ley de espectáculos públicos y actividades recreativas. La reforma pretende, además de corregir ciertos errores de la redacción original del reglamento, ampliar el número de eventos (espectáculos 0 actividades) que se pueden permitir, mediante autorización singular o comunicación previa (sujeta al cumplimiento de ciertas condiciones). La normativa citada trataba, a través de limitaciones sustantivas acústicas, horarias, de aforo, de tipo de actividad, etc., de conciliar los derechos de las personas organizadoras, espectadoras y del resto de la ciudadanía. Con esta reforma se suprime la referencia a que se consideren actividades recreativas habituales (y no ocasionales o puntuales, ni complementarias o accesorias) las celebraciones que superen el máximo de doce anuales, así como la prohibición de que puedan autorizarse más de dos en un mismo mes.

\section{MEDIDAS DE FOMENTO AMBIENTAL}

En el ámbito del Departamento de Medio Ambiente, Planificación Territorial y Vivienda se han establecido las bases reguladoras y convocado las siguientes medidas de fomento:

a) Subvenciones a entidades privadas que realicen proyectos para la generación de conocimiento en la conservación del patrimonio natural (Orden de 5 de junio de 2019, BOPV 116, 20 de junio).

b) Subvenciones destinadas a la compensación económica por la pérdida de rentabilidad financiera en la Reserva de la Biosfera de Urdaibai, y a su gestión medioambiental adecuada por medio de acuerdos de custodia (Decreto 83/2019, de 4 de junio, BOPV 108, 10 de junio).

c) Subvenciones a centros escolares no universitarios para el desarrollo del programa de Agenda 21 Escolar (Orden de 4 de junio de 2019, BOPV 107, 7 de junio). Esta disposición ha sido tomada conjuntamente con el Departamento de Educación. 
d) Subvenciones a empresas para la realización de inversiones destinadas a la protección del medio ambiente (Orden de 11 de junio de 2019, BOPV 114, 18 de junio).

e) Subvenciones a Ayuntamientos, Mancomunidades, otras Entidades Locales, Organismos Autónomos Locales, Agencias de Desarrollo Local y Sociedades Mercantiles Locales que realicen acciones que promuevan el desarrollo sostenible (Orden de 25 de junio de 2019, BOPV 127, 5 de julio).

f) Subvenciones a Ayuntamientos para la mejora de la información geográfica, realización de mapas municipales y su posterior difusión (Orden de 22 de mayo de 2019, BOPV 105, 5 de junio).

g) Subvenciones a Ayuntamientos, así como a los Concejos de los municipios alaveses, para la elaboración de planes de acción del paisaje (Orden de 18 de junio de 2019, BOPV 123, 1 de julio).

h) Subvenciones para la integración de la perspectiva de género en el planeamiento municipal y el urbanismo (Orden de 22 de mayo de 2019, BOPV 105, 5 de junio).

i) Ayudas del programa Eraikal para la implantación de sistemas de gestión, fomento de la calidad constructiva y arquitectónica y de la sostenibilidad y apoyo a la innovación de las empresas del sector de la edificación residencial (Orden de 9 de julio de 2019, BOPV 134, 16 de julio).

j) Ayudas del programa Renove en materia de rehabilitación eficiente de viviendas y edificios, para la elaboración de proyectos de intervención en el patrimonio edificado y la ejecucuión de obras derivadas de los mismos (Orden de 12 de junio de 2019, BOPV 116, 20 de junio).

Como función propia del Departamento de Desarrollo Económico e Infraestructuras, se han convocado las ayudas agroambientales a la diversificación de cultivos extensivos bajo técnicas de producción agrícola sostenible, previstas en el Programa de Desarrollo Rural de Euskadi 2015-2020 (Orden de 20 de junio de 2019, BOPV 123, 1 de julio), así como las ayudas a la paralización temporal de la actividad pesquera de la flota con puerto base en la 
CAPV, afectadas por el descanso biológico de la flota artesanal perteneciente al censo de artes menores en el Cantábrico Noroeste (Orden de 24 de junio de 2019, BOPV 135, 17 de julio). 